\title{
Using random walk models to simulate the vertical distribution of particles in a turbulent water column
}

\author{
André W. Visser* \\ Danish Institute for Fisheries Research, Department of Marine and Coastal Ecology, Kavalergården 6, DK-2920 Charlottenlund, \\ Denmark
}

\begin{abstract}
Random walk simulation has the potential to be an extremely powerful tool in the investigation of turbulence in environmental processes. However, care must be taken in applying such simulations to the motion of particles in turbulent marine systems where turbulent diffusivity is commonly spatially non-uniform. The problems associated with this nonuniformity are far from negligible and have been recognised for quite some time. However, incorrect implementations continue to appear in the literature. In this note computer simulations are presented to illustrate how and why these implementations are incorrect, and a simple technique that can properly simulate turbulent diffusion in the marine environment is discussed.
\end{abstract}

KEY WORDS: Random walk - Turbulence - Diffusivity Numerical simulation

Computer simulation of the movement of individual particles in a turbulent environment is becoming a much used and illuminating tool in investigating environmental processes. In the marine environment, processes that can be investigated in this way range from the distribution of planktonic cells and inter-particle encounter rates to the aggregation of organic material into flocs and marine snow, sedimentation rates and the trophic interactions of predators and their prey. The power of this method lies in its simplicity. Behavioural responses or history-dependent processes can be 'programmed' into the particle simulations in a transparent manner, and a variety of hypotheses can be tested.

The first and perhaps most crucial question to be addressed in such simulations is how individual particles move in response to turbulent diffusion. Here, a random walk model is most often used. The central premise of a random walk simulation is that, given a

\footnotetext{
•E-mail: awv@dfu.min.dk
}

diffusivity $K\left(\mathrm{~m}^{2} \mathrm{~s}^{-1}\right)$, the ensemble average (denoted by $\langle\ldots\rangle)$ of the square of the particle displacement $z$ is given by

$$
\frac{\mathrm{d}}{\mathrm{d} t}\left\langle z^{2}\right\rangle=2 K
$$

for a 1-dimensional process (e.g. Taylor 1921, Batchelor 1949, Csanady 1973, Berg 1983). For an individual particle, this translates to a change in position, from $z_{n}$ to $z_{n+1}$, over a finite time step, $\delta t$, given by

$$
z_{n+1}=z_{n}+R\left(2 r^{-1} K \delta t\right)^{1 / 2}
$$

where $R$ is a random process, with mean $\left\langle R^{2}\right\rangle=0$ and standard deviation $\left\langle R^{2}\right\rangle=r$. (e.g. if $R$ is a uniform distribution between +1 and -1 , then $r=1 / 3$ ).

It has long been recognised that turbulent diffusion in marine environments is not uniform. For instance, a water column on a shelf sea under the influence of wind, tides and stratification can exhibit a diffusivity profile which is high in the surface and bottom layers, and low in the vicinity of the pycnocline. An example of such a profile, calculated using a $k-\varepsilon$ turbulence closure scheme is shown in Fig. 1. If we begin with a uniform distribution of neutrally buoyant particles in this turbulent diffusive field, and calculate their redistribution by a straightforward (naive) random walk model, i.e.

$$
z_{n+1}=z_{n}+R\left[2 r^{-1} K\left(z_{n}\right) \delta t\right]^{1 / 2}
$$

then the particles eventually begin to accumulate in the region of low diffusivity. This is shown in Fig. 2, where maximum particle concentrations of 3 times the mean concentration are observed in the low diffusivity region after $6 \mathrm{~h}$ of simulation. If we equate the particles with phytoplankton cells, we might conclude that we have found an explanation for the subsurface chlorophyll maximum based purely on physical processes. However, the particles can just as well represent salt 


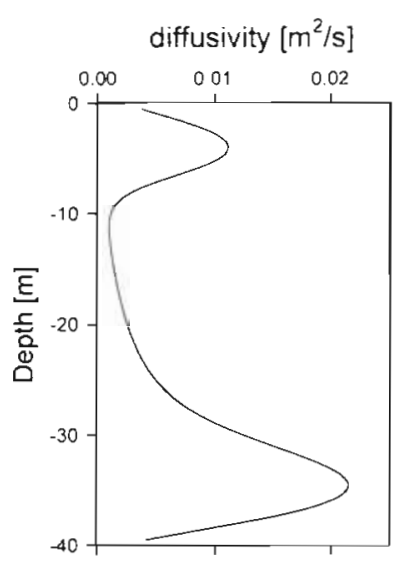

Fig. 1 A representative diffusivity profile as calculated by a $k-\varepsilon$ turbulence closure scheme as described in Burchard \& Baumert (1995). The water column depth is $40 \mathrm{~m}$. A surface wind stress corresponding to a wind speed of about $9 \mathrm{~m} \mathrm{~s}^{-1}$ is applied, as is a tidal current of amplitude $0.5 \mathrm{~m} \mathrm{~s}^{-1}$ There is moderate stratification: a vertical difference of $0.15 \mathrm{~kg} \mathrm{~m}^{-3}$ over a $15 \mathrm{~m}$ pycnocline separating vertically well-mixed surface and bottom layers

ions, in which case we have demonstrated that the salinity of the low turbulence region should increase. As pointed out by Holloway (1994), this is clearly incorrect.

In comparison, the equivalent situation described by a differential equation over the vertical co-ordinate $z$ and time $t$ for the particle concentration, $C$, may be written

$$
\frac{\partial C}{\partial t}-\frac{\partial}{\partial z}\left(K \frac{\partial C}{\partial z}\right)=0
$$

with boundary conditions

$$
K \frac{\partial C}{\partial z}=0
$$

at the surface and bottom. The steady state solution is $C(z)=C_{0}$ [i.e. $C(z)$ is constant], irrespective of the ver-

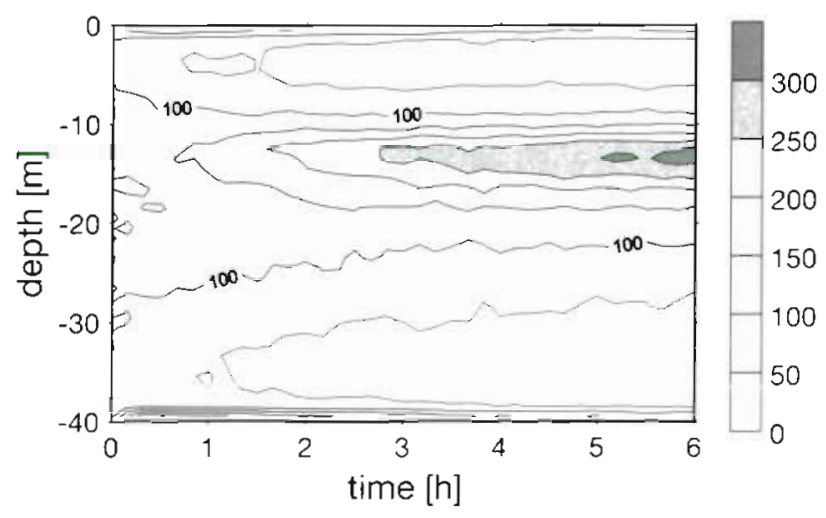

Fig. 2. Computer simulation of 4000 neutrally buoyant particles, initially distributed uniformly over depth, subject to the vertical diffusivity profile shown in Fig. 1. Subsequent trajectories are calculated using the naive random walk model, Eq. (3). Surface and bottom surfaces are reflecting; that is, if $z<-H$ then $z \rightarrow-(2 H+z)$ and if $z>0$, then $z \rightarrow-z$. The time step used is $6 \mathrm{~s} . R$ is a uniform random distribution over the interval $\left[+1,-1 \mid\right.$ giving $\langle R\rangle=0$ and $\left\langle R^{2}\right\rangle=1 / 3$. Results are presented as concentrations (particles $\mathrm{m}^{-1}$ ), and averaged over 10 min time intervals. $6 \mathrm{~h}$ of simulation are plotted. A perfectly uniform distribution would give 100 particles $\mathrm{m}^{-1}$ tical variation of $K(z)$, whether $C$ represents neutrally buoyant particles or any other passive tracer.

A resolution of these apparently inconsistent results can be inferred from careful consideration of the probability and/or moment statistics of the spatially varying diffusivity field. The formal aspects of these considerations have been well documented (e.g. Thomson 1984, Okubo 1986, Hunter et al. 1993). Following Hunter et al. (1993), it is demonstrated in Appendix 1 that the corrected random walk model corresponding to the situation described in the differential equation, Eq. (4), is

$z_{n+1}=z_{n}+K^{\prime}\left(z_{n}\right) \delta t+R\left\{2 r^{-1} K\left[z_{n}+1 / 2 K^{\prime}\left(z_{n}\right) \delta t\right] \delta t\right\}^{1 / 2}(6)$

where we use the shorthand notation, $K^{\prime}=\delta K / \delta z$, to represent the gradient of diffusivity. There are 2 differences between this, the diffusive random walk simulation, and the naive random walk, Eq. (3). Firstly, and most importantly, there is an additional nonrandom 'advective' component, $K^{\prime}\left(z_{n}\right) \delta$, from areas of low diffusivity to areas of high diffusivity. Secondly, the diffusivity is not estimated at the initial particle location, $z_{n}$, but offset a distance $1 / 2 K^{\prime}\left(z_{n}\right) \delta t$. Note also, that in the case where the diffusivity becomes uniform, $K^{\prime}(z)=0$, the naive and diffusive random walks become identical.

To illustrate the effect of these additional terms, we use the same diffusivity profile and situation as in Fig. 2 and calculate the redistribution of neutrally buoyant particles using the diffusive random walk model, Eq. (6). The results, presented in Fig. 3, clearly correspond much better with the differential equation solution; the distribution, apart from apparently random fluctuations no greater than $\pm 15 \%$ of the mean, remains virtually uniform. Apparently, the additional non-random term in Eq. (6) exactly balances the tendency of the naive model to collect particles in low diffusion regions.

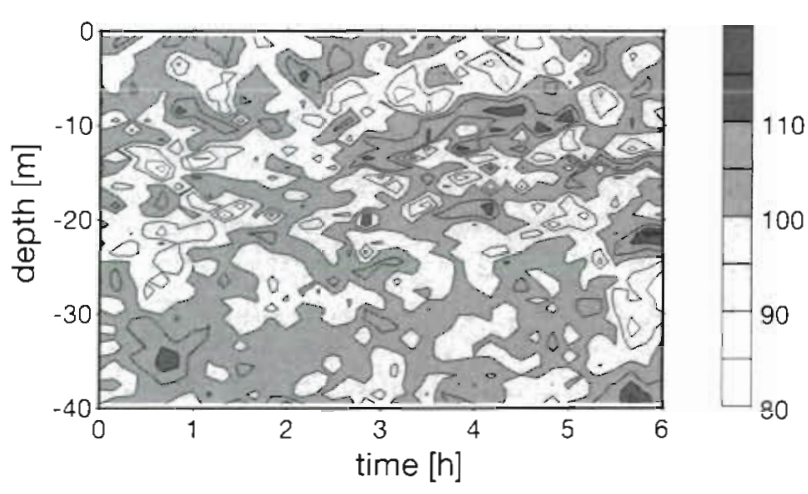

Fig. 3. As in Fig. 2 except that particle trajectories are simulated using the diffusive random walk model, Eq. (6). A perfectly uniform distribution would give 100 particles $\mathrm{m}^{-1}$ 
Most simply put, this term arises because, over a given time interval, particles at a particular location are influenced by slightly more energetic eddies originating in the area of high diffusion compared with somewhat less energetic eddies from the low diffusion region. Note that diffusivity is related to the turbulent velocity fluctuations, $w^{\prime}$, by $K=\left\langle w^{\prime 2}\right\rangle \tau$, where $\tau$ is the Lagrangian correlation time scale (cf. Csanady 1973). The effect of a gradient in the turbulent kinetic energy field is to induce a mean drift in the direction of increasing diffusivity (e.g. Legg \& Raupach 1982, Thomson 1984, Rahm \& Svenson 1986). This is sketched in Fig. 4. Suppose that a particle is at $z_{0}$, where diffusivity is $K_{0}$ and is locally non-uniform of the form $K(z)=K_{0}+$ $\left(z-z_{0}\right) K^{\prime}$. If we choose $R$ as a random step process of \pm 1 (i.e. $r=1$ ) then, in the first instance, the particle moves either up or down a distance $\delta z= \pm\left(2 K_{0} \delta t\right)^{1 / 2}$. However, the particle's upward motion is subject to slightly higher diffusivity (turbulent kinetic energy), $K^{+}=K_{0}+\left(2 K_{0} \delta t\right)^{1 / 2} K^{\prime}$. This gives a net upward displacement of

$$
\delta z^{+}=\left[2 K_{0} \delta t+2\left(2 K_{0} \delta t\right)^{1 / 2} K^{\prime} \delta t\right]^{1 / 2}=\delta z+K^{\prime} \delta t
$$

On the other hand, the particle's downward motion is subject to slightly lower diffusivity, $K^{-}=K_{0}-$ $\left(2 K_{0} \delta t\right)^{1 / 2} K^{\prime}$, which gives a net downward displacement of

$$
\delta z^{-}=\left[2 K_{0} \delta t-2\left(2 K_{0} \delta t\right)^{1 / 2} K^{\prime} \delta t\right]^{1 / 2} \approx \delta z-K^{\prime} \delta t
$$

The overall effect is that the centre of mass of the particles, initially at $z_{0}$, moves in the direction of increasing diffusivity at rate $K^{\prime}$.

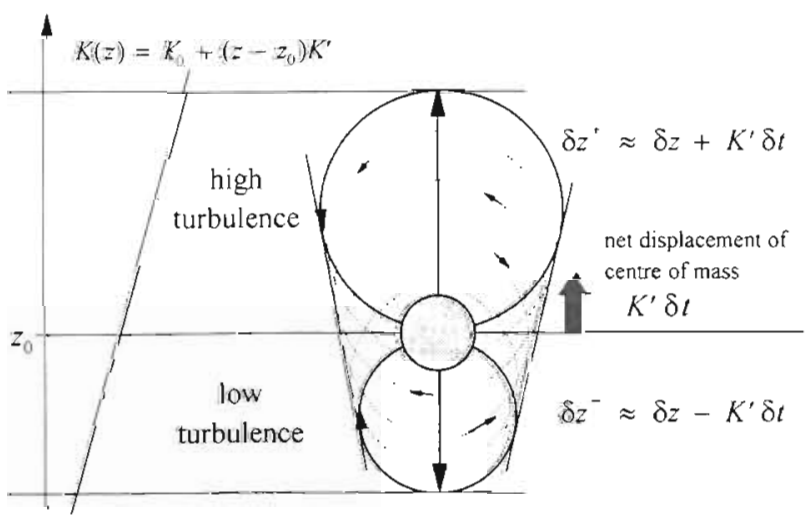

Fig. 4. Sketch of the eddies affecting the motion of a particle in a non-uniform diffusivity field. The maximum scale of the eddies in the vicinity of the particle is approximately $\delta z \approx$ $\left(2 K_{0} \delta t\right)^{1 / 2}$. However, because the turbulence field is nonuniform, eddies that sweep the particle upwards will be slightly larger than those that sweep it downwards. This disparity of maximum eddy scales introduces a net upward displacement, $K^{\prime} \delta t$, of the centre of mass of an ensemble average of particles initially at $z_{0}$
The principles outlined in the above paragraphs have been known for several years. Despite this however, either through a lack of knowledge or an underestimate of the importance of this effect, naive random walk models continue to appear in the literature applied to situations for which they are ill suited (e.g. Patterson 1991, Yamazaki \& Kamykowski 1991, Kamykowski et al. 1994, MacIntyre et al. 1995). While it is not evident what the implications of this erroneous application are for these particular studies, it is clear that there is a potential for quite substantial differences, as may be inferred by comparing Figs. $2 \& 3$. As is shown in Appendix 1, the naive random walk model of Eq. (3) corresponds to the differential equation

$$
\frac{\partial C}{\partial t}-\frac{\partial^{2}}{\partial z^{2}}(K C)=0
$$

with boundary conditions

$$
K \frac{\partial C}{\partial z}=0
$$

at the surface and bottom. This can be rewritten in the form

$$
\frac{\partial C}{\partial t}-\frac{\partial}{\partial z}\left(\frac{\partial K}{\partial z} C\right)-\frac{\partial}{\partial z}\left(K \frac{\partial C}{\partial z}\right)=0
$$

The second term in this equation represents an actual advection from areas of high diffusivity to areas of low diffusivity.

Admittedly, the random iterative process in Eq. (6) is an approximation, dependent ultimately on the size of the time step, $\delta t$. However, it should be stressed that Eq. (6) is not a higher order approximation to Eq. (3). That is, the discrepancy between the naive and corrected random walk models cannot be made negligibly small by decreasing the time step. In order to simulate particle motions over a finite time interval $\Delta t=n \delta t$, Eq. (6) must be iterated $n$ times, giving an advective displacement of $\sim K^{\prime} \Delta t$, irrespective of how small $\delta t$ is. The criterion for the appropriate application of Eq. (6) requires that, locally, the diffusivity be well approximated by

$$
K(z)=K_{0}+\left(z-z_{0}\right) K^{\prime}
$$

over the range of expected turbulent displacements, $z=z_{0} \pm\left(2 K_{0} \delta t\right)^{1 / 2}$. This means that higher order contributions to the Taylor expansion implied in Eq. (11) are small. Specifically, this suggests that the second derivative of diffusivity, $K^{\prime \prime}$, is such that

$$
K \gg 1 / 2\left[(2 K \delta t)^{1 / 2}\right]^{2} K^{\prime \prime}
$$

This criterion can always be met by choosing a small enough $\delta t$. In particular

$$
\delta t \ll \operatorname{MIN}\left(1 / K^{\prime \prime}\right)
$$


where MIN implies the minimum value over the region of interest. For the diffusivity profile in Fig. 1, this requires $\delta t \ll 300 \mathrm{~s}$.

A distinction should be made here between physical diffusion and 'biological' diffusion. For example, consider the rapid jump of a copepod which is based on a reaction to some environmental stimulus at a particular location. The length of the jump does not depend on the variation of the stimulus between the copepod's initial location and its end point. In this way, rapid jump behaviour can be modelled by a naive random walk so that behavioural diffusion can concentrate biota at particular locations

Lastly, we can consider the residence times of sinking particles in the turbulent (wind-mixed) surface layers of the ocean. As pointed out by Ruiz et al. (1996), there is an apparent paradox between the (naive) random walk model and intuition. In particular, if a particle has a settling velocity, $w$, and turbulence is simulated by a random upward or downward motion, then, on average, the random deflections by turbulence will average out and the effective settling velocity of the particles will remain unaffected. This is counter to the commonly held concept (cf. Ruiz et al. 1996) that increased mixing increases the residence time of particles in the mixed layer. Ruiz et al. (1996) examined the advection-diffusion equation for particle concentrations, $C(z)$. Here, increased residence times arise because turbulence decreases the flux, $F=C_{h} w$, out of the mixed layer, by decreasing $C_{\mathrm{h}}$ the particle concentration at the bottom of the mixed layer. In light of the diffusive random walk, we can now see how this increased residence time is brought about for individual particles; their effective settling velocity is in fact reduced at the bottom of the mixed layer where the gradient of diffusivity, $K^{\prime}$, can be large and directed upwards. That is, the centre of mass of a cloud of particles released at the base of the mixed layer will move towards the surface at a rate $K^{\prime}$. Ruiz et al. (1996) cite dynamics studies (Fung 1993, Wang \& Maxey 1993) which suggest the opposite. Namely, that for particles of low inertia (i.e. plankton), settling is essentially unaffected by turbulence. However, these resuits relate to isotropic turbulence, which is not the case at the bottom of the surface mixed layer.

As an illustration, Fig. 5 includes 3 examples of a random walk simulation for negatively buoyant particles $\left(w=-10^{-3} \mathrm{~m} \mathrm{~s}^{-1}=-86.4 \mathrm{~m} \mathrm{~d}^{-1}\right)$ under the influence of a turbulent diffusivity profile

$$
K(z)=K_{\grave{\partial}}-K_{\mathrm{b}} z \exp (\alpha z)
$$

corresponding to surface wind mixing. The first case, Fig. 5a, uses the naive random walk simulation Eq. (3), and the second case, Fig. 5b, uses the diffusive random walk Eq. (6). The third simulation case, Fig. $5 c$, is for a

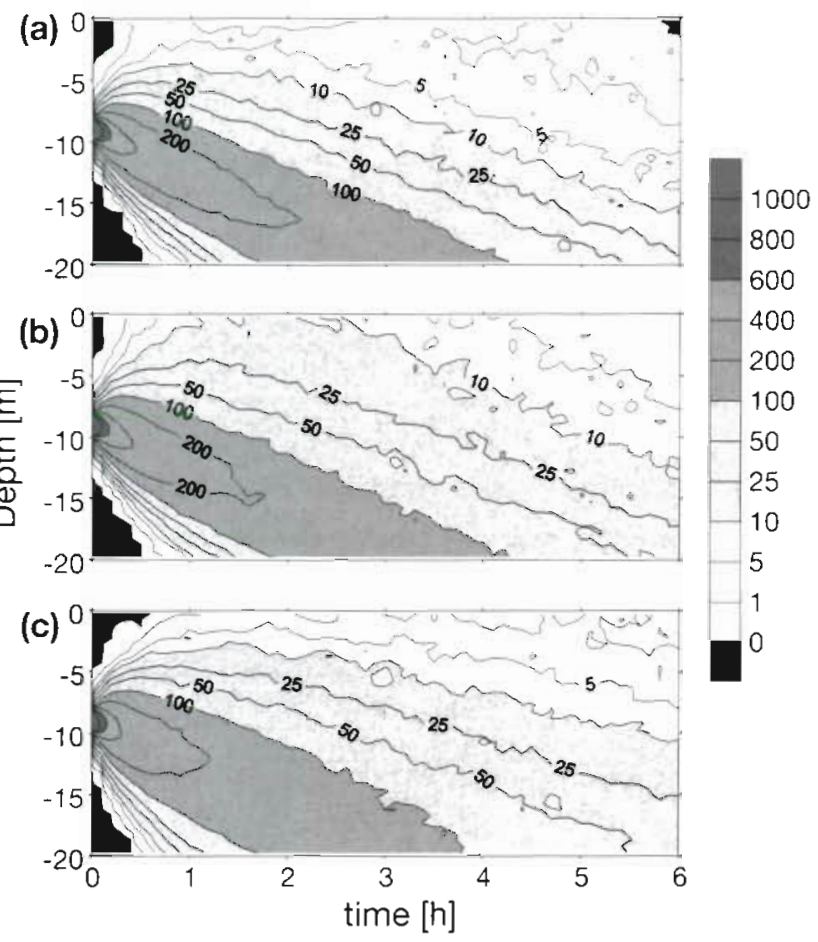

Fig. 5. Computer simulation of 4000 negatively buoyant particles ( $\left.w=-10^{-3} \mathrm{~m} \mathrm{~s}^{-1}=-86.4 \mathrm{~m} \mathrm{~d}^{-1}\right)$ initially distributed uniformly between 8 and $10 \mathrm{~m}$ depth subject to diffusivity $K(z)=$ $K_{\mathrm{a}}-K_{\mathrm{b}} z \exp (\alpha z)$, where $K_{\mathrm{a}}=10^{-3} \mathrm{~m}^{2} \mathrm{~s}^{-1}, K_{\mathrm{b}}=6 \times 10^{-3} \mathrm{~m} \mathrm{~s}^{-1}$, and $\alpha=0.5 \mathrm{~m}^{-1}$, for the (a) naive and (b) diffusive random walk simulations. Case (c) is for uniform diffusivity $K(z)=K_{c}=$ $2 \times 10^{-3} \mathrm{~m}^{2} \mathrm{~s}^{-1}$. The surface boundary is reflecting. The depth is effectively infinite, although for convenience it was not plotted below $20 \mathrm{~m}$. The time step used is $10 \mathrm{~s} . R$ is a uniform random distribution over the interval $[+1,-1]$. Results are plotted as concentrations (particles $\mathrm{m}^{-1}$ ), and averaged over 12 min time intervals

uniform diffusivity, $K_{c}$, equal to the average diffusivity over the upper $20 \mathrm{~m}$ of the water column. In each simulation, 4000 particles are released in a uniform distribution between 8 and $10 \mathrm{~m}$ depth. This is close to the base of the wind-mixed layer. The plots exhibit similar trends, with the densest concentrations of particles sinking out of the upper $20 \mathrm{~m}$ after 3 to $4 \mathrm{~h}$. This can be compared to the zero diffusion case where no particles would remain in the upper $20 \mathrm{~m}$ after $3.3 \mathrm{~h}$. In case (b), that for the diffusive random walk, it appears that a larger proportion of the particles reach higher into the surface water column, and remain there longer. than in either case (a) or case (c).

To underscore this, the total number of particles within the upper $5 \mathrm{~m}$ of the water column is plotted in Fig. 6. For brevity, this number of particles is denoted as $P_{\mathrm{a}}, P_{\mathrm{b}}$ and $P_{\mathrm{c}}$, for each case respectively. The number of particles for all cases reaches a maximum between 1 and $2 \mathrm{~h}$ after release. Subsequently, the 


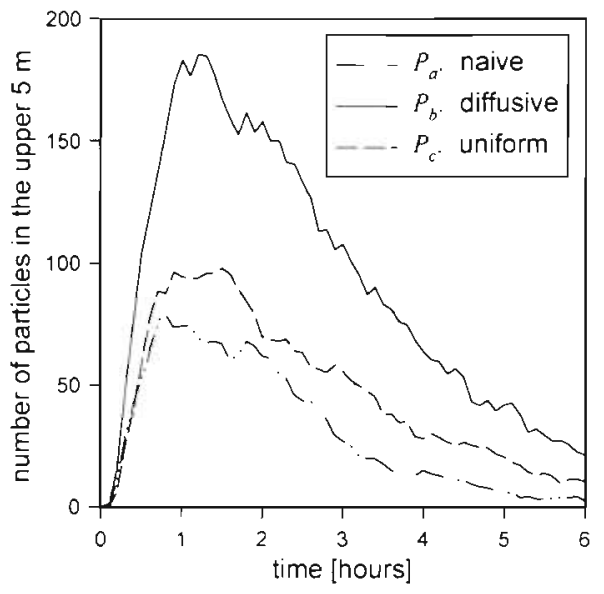

Fig. 6. Total number of particles within the upper $5 \mathrm{~m}$ of the water column for the 3 random walk simulations described in Fig. 5

number decreases with time in an apparent exponential decay. Comparing the naive $P_{\mathrm{a}}$ and uniform diffusivity $P_{c}$ shows that while their peak concentrations are about the same, the subsequent decrease in $P_{\mathrm{a}}$ is much faster than in $P_{\mathrm{c}}\left(P_{\mathrm{a}} \approx P_{\mathrm{c} / 2}\right.$ after $3 \mathrm{~h}, P_{\mathrm{a}} \approx P_{\mathrm{c} / 3}$ after $\left.6 \mathrm{~h}\right)$. From this, one would conclude that increasing the diffusivity in the surface mixed layer decreases the residence time of particles. On the other hand, the maximum concentration for the diffusive random walk $P_{\mathrm{b}}$ is about twice that for uniform diffusivity, i.e. $P_{\mathrm{b}} \approx$ $2 P_{\mathrm{c}}$. This difference of a factor of 2 persists throughout the subsequent decay period. The conclusion that can be drawn from this comparison is that the random walk model can simulate the increased residence times for particles in the surface mixed layer. Again we see that the diffusive random walk qualitatively agrees with conclusions drawn from differential equation solutions.

It is unfortunate that much of the literature surrounding random walk processes is posed in the spe- cialist mathematics of stochastic differential equations. While the rigour of these methods cannot be denied, they have tended to cloud important principles and processes (e.g. Yamazaki \& Kamykowski 1994), making them inaccessible to the non-expert user. However, as pointed out by Thomson (1987), the proof is in the pudding. Specifically, for a given physical situation, a stochastic model must converge with the differential equation solution over a sufficiently large number of realisations. If it doesn't, then the stochastic model is incorrect. In light of this criterion, this note has demonstrated that the naive random walk model Eq. (3) is systematically incorrect for non-uniform diffusivity. The correction required to make the random walk model consistent with the physical description is achieved quite simply, Eq. (6), without a drastic increase in computing costs.

Finally, it should be noted that physical models of turbulence are far from comprehensive. They can describe certain, but not all, aspects of turbulence. For instance, there is a serious shortcoming in physical models which lead to the parameterisation of the effects of turbulence as diffusivity. Such models, whether cast in terms of differential or random walk equations, can only say something sensible about the distribution of particles relative to a fixed co-ordinate frame. That is, in an Eulerian sense. The change in the relative distance between particles, i.e. a Lagrangian measure, is dependent on their separation (e.g. Csanady 1973). How this effect can be incorporated into a random walk model is far from clear.

It is hoped that this short note has clarified some aspects of random walk simulations so that they can be used to faithfully represent physical dispersion as is found in marine systems. This fidelity must be a fundamental requirement for integrated physical and biological models in order for their results to be meaningful.

Appendix 1

Suppose we have a region of space where the diffusivity is locally non-uniform and, with an appropriate choice of the $z$ origin, of the form $K(z)=K_{0}+z K_{0}^{\prime}$. We will examine the moments $M_{n}$ of the particle concentration $C$

$$
M_{n}=\int_{-\infty}^{\infty} z^{n} C \mathrm{~d} z
$$

for the two advection-diffusion like equations

$$
\begin{array}{ll}
\text { case }(\mathrm{a}): & \frac{\partial C}{\partial t}-\frac{\partial}{\partial z}\left(K \frac{\partial C}{\partial z}\right)=0 \\
\text { case }(\mathrm{b}): & \frac{\partial C}{\partial t}-\frac{\partial^{2}}{\partial z^{2}}(K C)=0
\end{array}
$$

Multiplying each equation by $z^{n}$ and integrating yields the local evolution of the $n$th moment. For example, case (a), Eq. (A.2a), gives

$$
\frac{\mathrm{d} M_{n}}{\mathrm{~d} t}=\int_{-\infty}^{\infty} z^{n} \frac{\partial C}{\partial t} \mathrm{~d} z=\int_{-\infty}^{\infty}\left(z^{n} K_{0} \frac{\partial^{2} C}{\partial z^{2}}+z^{n+1} K_{0}^{\prime} \frac{\partial^{2} C}{\partial z^{2}}+z^{n} K_{0}^{\prime} \frac{\partial C}{\partial z}\right) \mathrm{d} z
$$


Stipulating that the particle concentration remains spatially limited, that is both $C \rightarrow 0$ and $\partial C / \partial z \rightarrow 0$ as $z \rightarrow \pm \infty$, integration by parts yields:

$$
\frac{\mathrm{d} M_{n}}{\mathrm{~d} t}=K_{0} n(n-1) M_{n-2}+K_{0}^{\prime} n^{2} M_{n-1}
$$

From this, we can see that the oth moment, i.e. the total concentration, remains unchanged. The equation for the normalised moments $N_{n}=M_{n} / M_{0}$ then becomes

$$
\frac{\mathrm{d} N_{n}}{\mathrm{~d} t}=K_{0} n(n-1) N_{n-2}+K_{0}^{\prime} n^{2} N_{n-1}
$$

A similar treatment for case (b) leads to

$$
\frac{\mathrm{d} N_{n}}{\mathrm{~d} t}=K_{0} n(n-1) N_{n-2}+K_{0}^{\prime} n(n-1) N_{n-1}
$$

Setting the initial conditions of the distribution as a point source at $z=0$, i.e. $N_{0}=1, N_{1}=0, N_{2}=0$ at $t=0$, then integrating over a time interval, $(0, \delta t)$, gives the normalised moments of the distribution after time $\delta t$. In particular, case (a) leads to

$$
\begin{array}{ll}
\frac{\mathrm{d} N_{1}}{\mathrm{~d} t}=K_{0}^{\prime} & \Rightarrow N_{1}(\delta t)=K_{0}^{\prime} \delta t \\
\frac{\mathrm{d} N_{2}}{\mathrm{~d} t}=2 K_{0}+4 K_{0}^{\prime} N_{1} & \Rightarrow N_{2}(\delta t)=2 K\left[N_{1}(\delta t)\right] \delta t
\end{array}
$$

That is, after time $\delta t$, the centre of mass of the distribution, $N_{1}$, has moved a distance, $K_{0}^{\prime} \delta t$. Further, the second moment of the distribution, $N_{2}$, has increased by $2 K \delta$, where $K$ is evaluated at the distribution's new centre of mass.

In comparison, case (b) leads to

$$
\begin{array}{ll}
\frac{\mathrm{d} N_{1}}{\mathrm{~d} t}=0 & \Rightarrow N_{1}(\delta t)=0 \\
\frac{\mathrm{d} N_{2}}{\mathrm{~d} t}=2 K_{0}+2 K_{0}^{\prime} N_{1} & \Rightarrow N_{2}(\delta t)=2 K_{0} \delta t
\end{array}
$$

The centre of mass of the distribution remains unchanged, and its variance increases by $2 K_{0} \delta t$. In order for a random walk model to simulate either one of the differential equations ( $\mathrm{Eq}$. A2a, b), the random process involved should have the same moments as in the corresponding Eqs. (A.4) \& (A5). In particular, if $R$ is a random process with mean $\langle R\rangle=0$ and variance $\left\langle R^{2}\right\rangle$ $=r$, then the random walk simulations appropriate for each differential equation (Eq. A2a, b) are respectively

$$
\begin{array}{ll}
\text { case }(\mathrm{a}): & z_{n+1}=z_{n}+K^{\prime}\left(z_{n}\right) \delta t+R\left\{2 r^{-1} K\left[z_{n}+1 / 2 K^{\prime}\left(z_{n}\right) \delta t\right] \delta t\right\}^{1 / 2} \\
\text { case }(b): & z_{n+1}=z_{n}+R\left[2 r^{-1} K\left(z_{n}\right) \delta t\right]^{1 / 2}
\end{array}
$$

Thus, the naive random walk model, Eq. (A6b), simulates Eq. (A2b), which has an inherent advection term, $-\partial(C \partial K / \partial z) / \partial z$, in the direction of decreasing diffusivity. On the other hand, the diffusion equation, Eq. (A2a), is simulated using the diffusive random walk model, Eq. (A6a).

\section{LITERATURE CITED}

Batchelor GK (1949) Diffusion in a field of homogeneous turbulence. I Eulerian analysis. Aust J Sci Res 2:437-450

Berg HC (1983) Random walks in biology. Princeton Univer sity Press, Princeton

Burchard $H$, Baumert $H$ (1995) On the performance of a mixed-layer model based on the $k-\varepsilon$ turbulence closure. J Geophys Res 100:8523-8540

Csanady GT (1973) Turbulent diffusion in the environment. Riedel, Dordrecht

Fung JCH (1993) Gravitational settling of particles and bubbles in homogeneous turbulence. $J$ Geophys Res 98: $22645-22656$

Holloway G (1994) Comment: on modelling vertical trajectories of phytoplankton in a mixed layer. Deep Sea Res 41:957-959

Hunter JR, Craig PD, Phillips HE (1993) On the use of random walk models with spatially variable diffusivity. I Comput Phys 106:366-376
Kamykowski D, Yamazaki H, Janowitz GS (1994) A Lagrangian model of phytoplankton photosynthetic response in the upper mixed layer. J Plankton Res 16:1059-1069

Legg BJ, Raupach MR (1982) Markov-chain simulation of particle dispersion in inhomogeneous flows: the mean drift velocity induced by a gradient in Eulerian velocity variance. Boundary Layer Meteorol 24:3-13

MacIntyre S. Alldredge AL, Gotschalk CC (1995) Accumulation of marine snow at density discontinuities in the water column. Limnol Oceanogr 40:449-468

Okubo A (1986) Dynamical aspects of animal grouping swarms, schools, flocks, and herds. Adv Biophys 22:1-94

Patterson JC (1991) Modelling the effects of motion on primary production in the mixed layer of lakes. Aquat Sci 53:218-238

Rahm LA, Svenson U (1986) Dispersion of marked fluid elements in a turbulent Ekman layer. J Phys Oceanogr 16: $2084-2096$

Ruiz J, Garcia CM, Rodriguez J (1996) Sedimentation loss of 
phytoplankton cells from the mixed layer: effect of turbulence levels. J Plankton Res 18:1727-1734

Taylor GJ (1921) Diffusion by continuous movements. Proc Lond Math Soc 20:196-211

Thomson DJ (1984) Random walk modelling of diffusion in inhomogeneous turbulence. Q J R Meteorol Soc 110: $1107-1120$

Thomson DJ (1987) Criteria for the selection of stochastic models of particle trajectories in turbulent flows. J Fluid

Editorial responsibility: Otto Kinne (Editor),

Oldendorf/Luhe, Germany
Mech 180:529-556

Wang LP, Maxey MR (1993) Settling velocity and concentration of heavy particles in homogeneous isotropic turbulence. J Fluid Mech 256:27-68

Yamazaki H, Kamykowski D (1991) The vertical trajectories of motile phytoplankton in a wind mixed water column Deep Sea Res 38:219-241

Yamazaki H, Kamykowski D (1994) Reply to Greg Holloway Deep Sea Res 41:961-963

Submitted: January 21, 1997; Accepted: September 25, 1997 Proofs received from author(s): October 30, 1997 